\title{
Effective RSS Sampling for Forensic Wireless Localization
}

\author{
Yinjie Chen ${ }^{1}$, Zhongli $\mathrm{Liu}^{1}$, Xinwen $\mathrm{Fu}^{1}$, and Wei Zhao ${ }^{2}$ \\ 1 Computer Science Department, University of Massachusetts Lowell, \\ \{ychen1, zliu, xinwenfu\}@cs.uml.edu, \\ ${ }_{2}^{2}$ University of Macau, weizhao@umac.mo
}

\begin{abstract}
In many applications such as wireless crime scene investigation, we want to use a single device moving along a route for accurate and efficient localization without the help of any positioning infrastructure or trained signal strength map. Our experiments show that in a complicated environment, such as building corridors and downtown areas, triangulation or trilateration cannot be used for accurate localization via single device. A simple approach, which is better and robust, is to use where the maximum RSS (received signal strength) is sensed as the target's location. The question is how to make sure the maximum RSS is received while moving. Our novel RSS sampling theory presented in this paper answers this question: if RSS samples can reconstruct a target transmitter's power distribution over space, the location corresponding to the peak of such power distribution is the target's location. We apply the Nyquist sampling theory to the RSS sampling process, and derive a mathematical model to determine the RSS sampling rate given the target's distance and its packet transmission rate. To validate our RSS sampling theory, we developed BotLoc, which is a programmable and self-coordinated robot armed with a wireless sniffer. We conducted extensive simulations and real-world experiments and the experimental results match the theory very well. A video of BotLoc is at http://youtu.be/FsWLrH8Nj50.
\end{abstract}

\section{Introduction}

With the booming WiFi networks comes wireless network based crimes. One example explains this type of cyber crime scene well. According to the Huffington Post news on September 24, 2011 [30], law enforcement stormed into a Buffalo homeowner at 6:20 A.M, accusing him of sharing files for videos and images of children engaged in sexual acts. The fact is that his neighbor was using this unlucky man's open WiFi and committed the crime with a peer-to-peer file sharing software. At the end, the law enforcement released the unlucky man and arrested the real suspect after correlating logs of the involved peer-to-peer file sharing software from the offender and discovering his real ID. The Huffington Post news also gave example of similar crimes and other similar crimes were also reported elsewhere $[4,26,28,2,18]$. We also interviewed local state police, who confirmed such crimes have been occurring periodically. The Huffington Post 
news also cited survey result from the WiFi alliance, revealing that 32 percent of American people used a Wi-Fi network belonging to somebody else. This sheer fact explains why crimes over WiFi networks are widely spread. Further more, tech savvy criminals may also crack encrypted WiFi networks for crimes.

In this paper, we develop wireless network forensics techniques for fighting cyber crimes such as distribution of child pornography and cyber terrorism for public safety and homeland security. We target crime scenes of WiFi networks as discussed above, where people are mistakenly arrested for downloading child pornography. Such awkward moments not only bring much inconvenience to innocent people but also may alert real suspects, who may destroy the evidence to exonerate themselves. Not all investigators may be as lucky as the one in the Huffington Post news, who can use other online logs to identify the real suspect. To avoid such mishaps, an effective option for law enforcement is to use mobile and standalone WiFi sniffers, move around in the suspect region (driving a car or walking) and sense wireless signals transmitted from the target and locate its position. Such types of devices should be rapid in response, easy to operate, and have a universal use in various environments. Such a sniffer can be used in a legal way. Use the wireless crime scene investigation as an example. Before raiding the house, investigators can first acquire a search warrant or court order and use the WiFi sniffer to survey the suspect area and locate the real suspect. If necessary, another search warrant can be secured to search the real suspect. Please refer to Section 2 for further discussion of the legal matter.

We conduct localization experiments in a complicated environment, such as building corridors, and observe that, triangulation or trilateration often performs bad localization via single mobile device taking multiple measurements at different locations. Localization in other complicated environments such as downtown areas also produces poor localization accuracy. A simple approach for a single device locating a target is to use where the maximum RSS is sensed as the target's location. Therefore, a question to answer is how to make sure the maximum RSS is received while moving. Astute readers may answer this question instantly: use over-sampling and move slowly to collect as many RSS samples as possible. However, what do we mean by over-sampling? How slow is slow? We will address these fundamental issues in this paper.

We propose the RSS sampling theory to address the problem of sampling: if RSS samples can reconstruct a target transmitter's power distribution over space, the location corresponding to the peak of such power distribution is the target's location. Our RSS sampling theory explains the relationship between the sniffer's velocity, target traffic pattern, and the distance between the target and sniffing route.

We implemented a prototype system, BotLoc, to demonstrate the usability of our RSS sampling theory. BotLoc is a programmable and self-coordinated robot (P3-DX [14]) armed with a wireless sniffer and it can be used for both indoor and outdoor localization. We introduce a set of localization schemes powered by the RSS sampling theory for BotLoc. With an appropriate velocity, the robot correctly collects RSS samples along its route and is able to derive the maximum 
RSS at the intersection point of the route and its perpendicular line through the target. Using this method, we can determine the angle of the target with respect to the route.

Our major contributions can be summarized as follows:

- We are the first to propose the RSS sampling theory for a moving sniffer, which can be carried by humans or vehicles, to reconstruct the target power distribution via a sampled RSS time series. We demonstrate the use of this RSS sampling theory via precise localization using a moving robot. We proposed a set of general theories to enhance the accuracy and efficiency of wireless mobile localization in this context. We explicitly formulated the relationship between the robot's velocity, the target wireless device's packet transmission interval, and the distance between the target and robot's route. This RSS sampling theory guarantees sufficient RSS collection for precise localization and avoids over-sampling, or in other words, answers what is over-sampling.

- We developed a fully functional localization system: BotLoc. BotLoc is a P3-DX robot armed with a wireless sniffer. It applies our RSS sampling theory for collecting RSS samples and locating a target mobile device. It is infrastructure-free and training-free. Our contribution also includes extensive experimental results which demonstrate the effectiveness of the proposed theorem.

The rest of this paper is organized as follows. Section 2 introduces our motivation to develop RSS sampling theory for forensic localization in a wireless network crime scene investigation and related legal issues. Section 3 presents the application background, problem definition of RSS sampling and the solution, the RSS sampling theory. Section 4 introduces the BotLoc system for localization and presents experimental evaluation of BotLoc. Section 5 discusses related work, and Section 6 concludes this paper.

\section{Motivation and Legal Issues}

As we discussed in the introduction, the pervasive deployment of WiFi has provided an easy venue for cyber criminals to commit crimes including accessing illegal content anonymously $[4,26,28,30,2,18]$. Another interesting case is that experienced hackers also utilize public WiFi network to commit crimes. The notorious hacker, Max Butler, who was sentenced to 13 years in prison [22] in February 2010, often stayed at a large hotel in downtown San Francisco and used open wireless networks or hacked wireless networks with weak encryption to commit remote attacks while hiding behind wireless routers.

The difficulty for law enforcement is that, as the network address translation technique (NAT) is widely used in wireless routers, the observed public IP address may not reveal the real location information of the criminals. Therefore, law enforcement is not able to tell wether the criminal is in the house which is associated with the observed IP address, or in a neighbour's house. Moreover, 
without evidence to prove that the criminal is a neighbour, law enforcement can not get a search warrant to carry out the search in that neighbour's house. To address this problem, Yang et al. [32] proposed methods to remotely identify whether a criminal is using wireless or wired network while committing crimes. If the criminal is using wired network to commit crimes, then the criminal should be in the house where the observed public IP address is associated. As the authors acknowledge, the robustness and effectiveness of their approach need further improvement.

To the best of our knowledge, currently there is no effective and efficient technique to locate a criminal which is committing crimes using a wireless network. The best solution is to develop a forensic localization tool for wireless network crime scene investigation. This tool could help law enforcement to collect evidence about criminals' location. From an interview with local state police, we have confirmed that law enforcement urgently needs such a localization toolkit, and requires the toolkit to be portable, infrastructure-free, and not reliant on any training data such as a trained RSS map. Besides, forensic localization using such toolkit should not violate the law.

To conduct forensic localization legally, the localization toolkit should be used in the following way. First of all, the law enforcement identifies illegal activities that are coming from a public IP address which is associated to a house A. Take the peer-to-peer file sharing of child pornography as an example. The investigators download child pornography from a peer and acquires the associated IP address. If the IP address is associated with a broadband connection rather than a cellular connection, an ISP's billing record can be subpoenaed to recover a physical address [32]. Secondly, law enforcement obtains authorization, a wiretap, to monitor the activities of A's router, and derives MAC address of the target machine that is performing illegal activities. Thirdly, the law enforcement uses a forensic localization toolkit to collect only wireless signal strength information which is related to that target machine. This localization requires legal authorization such as a subpoena or a court order. To collect necessary wireless traffic in order to perform the localization, law enforcement can download the illegal content from the suspect computer using the peer-to-peer file sharing software. Finally, after the target computer is located, law enforcement obtains a search warrant and searches the suspect's computer.

In the rest of this paper, we focus on how to collect wireless traffic and locate the suspect.

\section{RSS Sampling}

We devote this section to the problem of RSS sampling because of its importance in various application scenarios. We will first discuss the application background, then define the problem of RSS sampling and finally present the solution. 


\subsection{Background}

As we mentioned in the introduction, a simple approach for a single device locating a target is to use where the maximum RSS is sensed as the target's location. We will propose the RSS sampling theory to address the problem of sampling: if RSS samples can reconstruct a target transmitter's power distribution over space, the location corresponding to the peak of such power distribution is the target's location where we sense the maximum RSS from the target.

Since we are concerned with RSS distribution over space, we now introduce a wireless propagation path loss model in $(1)[8,6]$. It gives the relationship between the distance and RSS at a receiver,

$$
P(d)=P(1)-10 \alpha \log (d)-W+X \sigma,
$$

where distance $d$ (in meters) is the receiver-transmitter distance and power $P(d)$ is the RSS at the receiver's antenna. $\alpha$ is the path loss exponent, $W$ (in $\mathrm{dB}$ ) is the wall attenuation degree, and $X \sigma$ is a normally distributed variable with mean of 0 and variance of $\sigma^{2} . X_{\sigma}$ is caused by phenomena including multipath propagation. This $\log$ normal wireless propagation model is merely an approximation. We find this approximation is accurate enough to address our problem.

\subsection{The Problem}

The RSS sampling problem is defined as follows. A moving wireless sniffer, carried by a vehicle or human, moves along a route and collects RSS samples (a RSS map) along a route. The moving velocity is adjustable. How can we sample RSS so that these RSS samples can be used to reconstruct the target's transmission power distribution over the route? This simple scenario catches the essence of the sampling problem and also has its own application, such as surveillance along a corridor.

Without loss of generality, we use a moving robot equipped with a wireless sniffer to explain the RSS sampling process. A robot is used because it is self coordinated and its speed measurement is simple and stable with accompanying robot APIs. Figure 1 shows an example of the target power distribution $s(t)$ over a route. Those dots below the curve $s(t)$ represent RSS samples collected by the robot armed with a sniffer. The target's orthogonal projection onto the route is denoted as the origin $O$. An extreme counter-example is that if the robot is running 100 meters per second and the target is transmitting 1 packet per second, we cannot reconstruct the target power space distribution $s(t)$ along the route because there are too few RSS samples. In reality, it is highly possible that the packet transmission rate of a target (e.g. a laptop) may be quite slow. Hence, a strict control of the moving velocity is necessary.

In practice, $s(t)$ can be much more complicated because of the multipath effect in an indoor environment. The theories presented in this paper actually give upper bounds of the robot velocity for reconstructing $s(t)$. Our experimental results in Section 4 match the theory very well. 


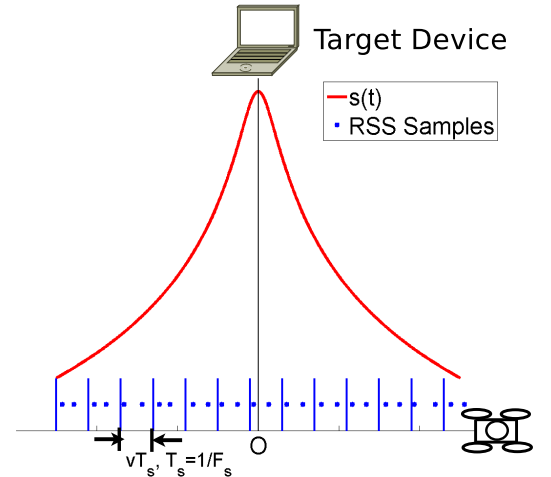

Fig. 1. Power Distribution s(t) Over a Route

\subsection{Our Solution}

We now present a fundamental theorem for a moving sniffer to correctly collect RSS samples, with which we can reconstruct the target's power distribution. For clarity and conciseness, we assume that a robot carries the sniffer and such a system produces a moving sniffer.

Recall Formula (1) gives the physical model of wireless signal attenuation. We define $s(t)$ as the power distribution over a route. In the discussion of RSS sampling, we ignore the noise term $X \sigma$ in Formula (1). This does not affect the essence of our sampling theory. Furthermore, noise is of high frequency and the sampling process filters a part of the noise.

We use the signal sampling theory [1] to address the problem. Considering the velocity of the robot and the target device packet transmission rate, we derive a RSS sampling theorem in Theorem 1.

Theorem 1. A robot armed with a wireless sniffer moves at a velocity of $v \mathrm{~m} / \mathrm{s}$ along a route. The target wireless device transmits at a rate of $F_{P k t}$ packets per second. The collected RSS samples can be used to reconstruct the target power distribution along the route if and only if the following condition is satisfied,

$$
v<\frac{F_{P k} v_{0}}{2 F_{0}},
$$

where $v_{0}$ is a baseline velocity, and $F_{0}$ is the bandwidth of $s(t)$ at velocity $v_{0}$, which is denoted as $s_{0}(t)$.

We use Figure 1 to explain how we prove Theorem 1. Recall $s(t)$ is the target power distribution $s(t)$ over the route, dots below $s(t)$ are RSS samples, and the origin $O$ is the target's orthogonal projection on the route. We refer to the Nyquist sampling theorem and claim that, in order to reconstruct $s(t)$, the RSS sampling frequency $F_{s}$ should be higher than twice of $s(t)$ 's band limit $F_{\max }$. Based on this point, it is obvious that the sniffer should sample in every space interval of length $v T_{s}$ along the route, where $T_{s}=\frac{1}{F_{s}}$ is the sampling interval. In Figure 1, we place a set of short bars as boundaries of the space intervals along the route. The sniffer should collect at least one packet within each interval. Therefore, the robot should control its velocity and stay within each cell longer 
than the packet transmission interval. Since $s(t)$ scales over speed $v$, so does $F_{\max }$ vary over $v$. In order to represent $F_{\max }$ using speed $v$, we use Fourier transform's properties, select $v_{0}$ as a baseline velocity, and build the relationship among $F_{0}, v_{0}, F_{\max }$ and $v$, where $F_{0}$ is the bandlimit of $s(t)$ at speed $v_{0}$. Finally, we have Formula (2). The proof of Theorem 1 can be found in Appendix A of our technical report which is available on requirement.

Theorem 1 tells us that in order to balance accuracy and efficiency, we should control our robot to move reasonably fast, but below a velocity of $\frac{v_{0} F_{P k t}}{2 F_{0}} \mathrm{~m} / \mathrm{s}$.

\section{Evaluation}

We conducted extensive experiments and simulations to verify the correctness of our RSS sampling theory. In this section, we first introduce our system BotLoc, a moving robot armed with a wireless sniffer for localization. Then, we present experimental results to validate our RSS sampling theory.

\subsection{BotLoc System}

Figure 2 shows the architecture of BotLoc while Figure 3 shows a photo of the BotLoc prototype. BotLoc consists of two main components: (i) robot subsystem, and (ii) positioning subsystem.

The robot subsystem drives the robot along a specified route by accepting velocity and heading commands from a software controller on a laptop, which steers the robot. The robot has an odometry coordinate system and returns its odometric pose to the controller program. The pose is represented as $(x, y, \theta)$, where $x$ and $y$ are the two-dimensional coordinates, and $\theta$ is the orientation of the robot with respect to its starting pose.

The positioning subsystem utilizes an antenna to sniff wireless packets and derive RSS samples. The localization analyzer correlates the collected RSS to the robot's location over time. Therefore, when the surveillance is finished, the localization analyzer inspects the signal strength time series coupled with position information in order to compute the target's location. Once the computation is finished, the positioning subsystem sends the result to the robot subsystem via the software controller, which drives the robot to the derived position. During the surveillance operation, the positioning system may also command the robot to adjust its speed and heading and cater to the target's transmission pattern. We now introduce BotLoc's configuration. The robot we choose is $P i$ oneer P3-DX [16], a remotely operated and programmable robot. P3-DX can be controlled by either a joystick or a program. The robot is assembled with a laser rangefinder (LMS200 [15]), a sonar array, and installed with a laser mapping and navigation system, which enables the robot to map the environment such as a building floor. The robot controller runs on a Lenovo W500 laptop, and it uses an object-oriented, robot control applications-programming interface (ARIA) from the manufacturer to control the robot. A robot operator can 


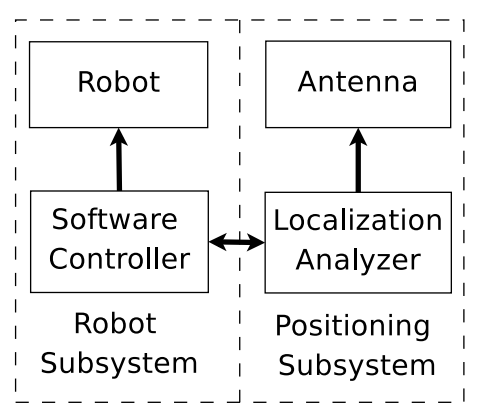

Fig. 2. BotLoc Function Modules

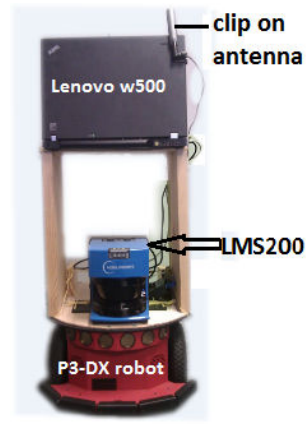

Fig. 3. Prototype

launch a client program such as MobileEyes [14] to set up a TCP connection to the robot controller, and execute localization tasks remotely.

The positioning subsystem uses a Ubiquiti 802.11a/b/g PCMCIA Cardbus connected to a clip-on antenna and runs a wireless sniffing program on the laptop. If we need to generate a map with AP positions marked, Kismet can collect wireless traffic of the whole spectrum via channel hopping.

\subsection{Experiment Setup}

We design real world experiments in two different environments: office, and track field.

In the office case, we conduct indoor localization. We place a laptop in one office room as a target, which keeps sending out ICMP packets at a constant rate of 100 packet/s. We drive BotLoc along the corridor along a straight line, and choose the position where the peak value of the target's power distribution is sensed to be the target's estimated position. Although this estimated position is not accurate enough to point out where the target stays in the room, this result is sufficient for us to illustrate the accuracy of our localization scheme via applying the RSS sampling theory. To determine the bound of robot's velocity, we calculate the target's average packet transmission rate, which is 111 packet/s. This rate is higher than 100 packet/s that we set to the target, because in the monitor mode, the sniffer is able to capture the re-transmitted frames.

To demonstrate how the robot's velocity affects localization accuracy, we synthesize multiple robot pose time series under different robot speeds, and calculate the estimated projection of targets using the synthesized data. With a group of robot pose time series collected from real-world experiments, our simulation follows four steps: (i) We select a group of velocities. (ii) For each velocity, we synthesize new robot pose time series from our real-world data, respectively. (iii) With synthesized pose time series and RSS time series, we use linear interpolation to identify the location with the maximal RSS readings. (iv) We determine the estimated projection point $X^{\prime}$. We set velocity $v$ to be $0.2,2$, $20,40.0,80.0,160.0,320.0,640.0,1280.0$, and $2560.0 \mathrm{~m} / \mathrm{s}$, and run simulations for each value. The simulation results are presented in Figure 4. 
In the case of track field, we conducted outdoor localization via the triangulation principle to actually derive the target's position. First, we choose a Nexus One smartphone as our target. We set the smartphone as an AP so that it keeps broadcasting beacon frames. Next, we design two perpendicular lines as our routes. We place the smartphone 1 meter away from both routes, and locate it via BotLoc. Then we draw two perpendicular lines to these routes with each line passing through the position where the maximum RSS is sensed along a route. The intersection of these two perpendicular lines is chosen as the target's position $X^{\prime}$. Therefore, the localization error is the distance from this intersection $X^{\prime}$ to the target's real location. We adjust the distance between the smartphone and each of the routes to be 1,2, 4, 8, 12 meters, and test 20 times for each distance respectively.

\subsection{Performance of BotLoc}

In the office case, we present our localization experiment results in Figure 4. Figure 4 shows that (i) when the velocity is below $320 \mathrm{~m} / \mathrm{s}$, the average distance error is below two meters. This cutoff velocity is large because the target is transmitting very fast at 111 packets/s. It also explains why related work [11], [24] can derive seemingly correct results although they don't have the support of our RSS sampling theorem; (ii) The localization error changes with the robot velocity. These observations validate our RSS sampling theorem.

In the track field case, we present our localization experiment results via the triangulation strategy in Figure 5 . The $x$-axis is the distance between the target and each route, while the $y$-axis is the localization error under each setting. The solid line represents the mean of localization error and the short segments show the confidence intervals with respect to every mean. As the distance increases, the confidence interval grows, too. When the distance is 12 meters, the mean is 2.4 meters. This is acceptable as it is easy for human beings to spot a mobile device within a range of 2.4 meters nearby.

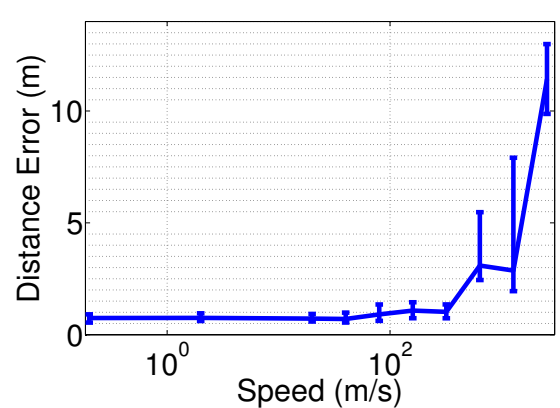

Fig. 4. Localization Error vs. Velocity

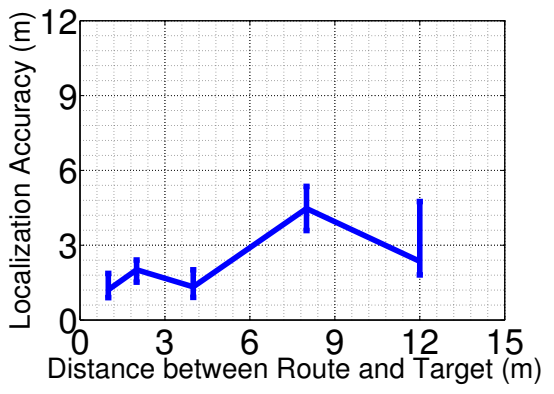

Fig. 5. Localization Accuracy with Triangulation 


\section{Related Work}

There has been extensive work on device positioning in WiFi and sensor networks. Due to space limitations, we only review the existing work most related to our paper: Most existing techniques provide localization in a two-dimensional space (e.g., longtitude/latitude). Positioning systems are classified as outdoor and indoor systems, respectively, which feature vastly different requirements and techniques. The most popular outdoor positioning system is GPS [7]. Many cellular mobile networks also belong to this category, and allow the tracking of powered-on devices through the operator's base-transceiver stations. Indoor positioning systems include RADAR [3], LANDMARC [20], the digital Marauder's Map [10], Lighthouse [25], and VORBA [21]. All these systems position a mobile device based on the measured signal strength. In particular, the former three utilize a dense grid of omnidirectional base stations, while the latter two rely on base stations with revolving unidirectional antennas. Active Badge [31], Active Bat [13] and Cricket [23] can provide better localization accuracy than outdoor systems due to the usage of a large number of positioning-support sensors.

Subramanian et al. [27] use electronically steerable Phocus Array antennas from Fidelity Comtech [9] for wardriving and collecting RSSs with directional information. Han et al. [12] take RSS samples from wardriving and use the gradient information derived from RSS to infer the position of an AP.

The indoor localization technique using smartphones is widely studied by researchers. Point Inside [17] and Google Maps for Android [19] are smartphone applications developed for malls, retailers, and airports. Point Inside helps navigate users to stores or products of interest. Google Maps for Android shows a user his or her location on an indoor map. [29] utilizes acoustic background spectrum $(\mathrm{ABS})$ as a room fingerprint to determine which room a smartphone holder visits. Quigley et al. [24] leverage robots to generate indoor maps with off-the-shelf SLAM techniques and use multiple sensors to collect training data including WiFi signals, camera images, and locate a human by searching for closest matched training data. Constandache et al. [5] creatively adopt audio beacons to navigate people via mobile phones. They capture users' movement traces using accelerometer and compass measurements and establish a global view of users' positions in a remote server. This server provides routes to users to reach the people of interest.

\section{Conclusion}

In this paper, we have addressed a critical, but unanswered question in a forensic wireless localization scene: How can we use collected RSS samples by a moving sniffer, carried by humans or vehicles, to reconstruct the target transmitter's power distribution in the space? With a reconstructed RSS distribution, we can derive the location yielding the maximum RSS. Our experiments show that in a complicated environment such as building corridors, triangulation cannot be used for accurate localization via single device. A simple approach, which is 
better and more robust, is to use where the maximum RSS is sensed as the target's location.

We innovatively apply the Nyquist sampling theory to address this problem and develop our RSS sampling theory. We demonstrate the use of this RSS sampling theory via precise localization using a moving robot. We developed a fully functional localization systems: BotLoc. BotLoc is a P3-DX robot armed with a wireless sniffer. It applies our RSS sampling theory for collecting RSS time series and locating a target mobile device with a moving robot armed with a wireless sniffer. It is infrastructure-free and training-free. Our contribution also includes extensive experimental results which demonstrate the effectiveness of the proposed theorem and systems. We expect that our theory and system provide a fundamental empirical and theoretical framework for forensic wireless localization via moving sniffers.

\section{References}

[1] J. R. B. A. V. Oppenheim, R. W. Schafer. Discrete-time signal processing. In Prentice Hall, 1999.

[2] N. Anderson. Swat team throws flashbangs, raids wrong home due to open wifi network. http://arstechnica.com/tech-policy/2012/06/ swat-team-throws-flashbangs-raids-wrong-home-due-to-open-wifi-network/, 2012.

[3] P. Bahl and V. N. Padmanabhan. RADAR: An in-building RF-based user location and tracking system. In Proceedings of INFOCOM, 2000.

[4] C. Chan. The swat team gently reminds a girl to secure her wi-fi network by raiding her house with flashbangs. http://gizmodo.com/ 5922322/the-swat-team-gently-reminds-a-girl-to-secure-her-wi+ fi-network-by-raiding-her-house-with-flashbangs, 2012.

[5] I. Constandache, X. Bao, M. Azizyan, and R. R. Choudhury. Did you see bob?: human localization using mobile phones. In Proceedings of the sixteenth annual international conference on Mobile computing and networking, 2010.

[6] G. D. Durgin, T. S. Rappaport, and H. Xu. Measurements and models for radio path loss and penetration loss in and around homes and trees at $5.85 \mathrm{ghz}$. ACM Transactions on Communications, 46(11):1484-1496, November 1998.

[7] P. Enge and P. Misra. Special issue on global positioning system. Proceedings of the IEEE, 87(1):3-15, January 1999.

[8] D. B. Faria. Modeling signal attenuation in ieee 802.11 wireless lans - vol. 1. In Stanford University, Tech. Rep., July 2005.

[9] Fidelity Comtech, Inc. 802.11 phocus array antenna system by fidelity comtech. http://www.fidelity-comtech.com/, 2009.

[10] X. Fu, N. Zhang, A. Pingley, W. Yu, J. Wang, and W. Zhao. The digital marauders map: A new threat to location privacy in wireless networks. In Proceedings of ICDCS, 2009.

[11] A. Haeberlen, A. Rudys, E. Flannery, D. S. Wallach, A. M. Ladd, and L. E. Kavraki. Practical robust localization over large-scale 802.11 wireless networks. In in Proceedings of the 10th Annual International Conference on Mobile Computing and Networking (MOBICOM, 2004. 
[12] D. Han, D. G. Andersen, M. Kaminsky, K. Papagiannaki, and S. Seshan. Access point localization using local signal strength gradient. In Proceedings of Passive E Active Measurement (PAM), 2009.

[13] A. Harter, A. Hopper, P. Steggles, A. Ward, and P. Webster. The anatomy of a context-aware application. In Proceedings of MOBICOM, 1999.

[14] A. T. Inc. Mobileeyes. http://www.mobilerobots.com/ResearchRobots/ PioneerSDK/MobileEyes.aspx, 2011.

[15] A. T. Inc. Laser navigation package. http://www.mobilerobots.com/ ResearchRobots/Accessories/LaserNavigationPkg.aspx, 2012.

[16] A. T. Inc. Pioneer p3-dx. http://www.mobilerobots.com/researchrobots/ researchrobots/pioneerp3dx.aspx, 2012.

[17] P. I. Inc. Pointinside. http://www.pointinside.com/, 2012.

[18] KTRK-TV/DT. Man accused of downloading child porn over his neighbor's wi-fi. http://abclocal.go.com/wabc/story?section=news/local\&id=8917541, 2012.

[19] B. McClendon. A new frontier for google maps: mapping the indoors. http://googleblog.blogspot.com/2011/11/ new-frontier-for-google-maps-mapping.html, 2012.

[20] L. M. Ni, Y. L. Yiu, C. Lau, and A. P. Patil. LANDMARC: Indoor location sensing using active RFID. In Proceedings of PerCom, pages 407-415, 2003.

[21] D. Niculescu and B. Nath. VOR base stations for indoor 802.11 positioning. In Proceedings of MOBICOM, 2004.

[22] K. Poulsen. Record 13-year sentence for hacker max vision. http://www.wired. com/threatlevel/2010/02/max-vision-sentencing/, 2010.

[23] N. B. Priyantha, A. Chakraborty, and H. Balakrishnan. The Cricket LocationSupport System. In Proceedings of MOBICOM, 2000.

[24] M. Quigley, D. Stavens, A. Coates, and S. Thrun. Sub-meter indoor localization in unmodified environments with inexpensive sensors. In IROS, pages 2039-2046. IEEE, 2010.

[25] K. Römer. The lighthouse location system for smart dust. In Proceedings of MobiSys, 2003.

[26] s.e. Jones. Internet access stealing becoming a major law enforcement concern. http://voices.yahoo.com/ internet-access-stealing-becoming-major-law-enforcement-11216494, 2012 .

[27] A. P. Subramanian, P. Deshpande, J. Gao, and S. R. Das. Drive-by localization of roadside wifi networks. In Proceedings of INFOCOM, 2008.

[28] A. Sylvain. Internet thieves piggyback on legitimate users. http://www . usatoday . com/tech/news/story/2012-04-08/internet-theft-web/54116488/1, 2012.

[29] S. P. Tarzia, P. A. Dinda, R. P. Dick, and G. Memik. Indoor localization without infrastructure using the acoustic background spectrum. In Proceedings of the 9th international conference on Mobile systems, applications, and services, 2011.

[30] C. Thompson. Innocent man accused of child pornography after neighbor pirates his wifi. http://www.huffingtonpost.com/2011/04/24/ unsecured-wifi-child-pornography-innocent_n_852996.html, 2011.

[31] R. Want, A. Hopper, V. Falcao, and J. Gibbons. The active badge location system. ACM Transactions on Information Systems, 10(1), January 1992.

[32] S. Yang, J. Kurose, and B. N. Levine. Disambiguation of residential wired and wireless access in a forensic setting. In Proceedings of INFOCOM, 2013. 